\title{
Article \\ Zinc Finger-Homeodomain Transcriptional Factors (ZF-HDs) in Wheat (Triticum aestivum L.): Identification, Evolution, Expression Analysis and Response to Abiotic Stresses
}

\author{
Hao Liu ${ }^{1,2,+} \mathbb{C}$, Ying Yang ${ }^{3,+}+\mathbb{D}$ and Linsheng Zhang ${ }^{1, *}$ \\ 1 College of Life Science, Northwest A\&F University, Yangling 712100, China; liuhao_1990@126.com \\ 2 College of Agriculture, Ludong University, Yantai 264011, China \\ 3 College of Nursing, Weinan Vocational \& Technical College, Weinan 714000, China; yy820923@sina.com \\ * Correspondence: linszhang@nwsuaf.edu.cn \\ + These authors contributed equally to this work.
}

check for updates

Citation: Liu, H.; Yang, Y.; Zhang, L. Zinc Finger-Homeodomain Transcriptional Factors (ZF-HDs) in Wheat (Triticum aestivum $\mathrm{L}$.): Identification, Evolution, Expression Analysis and Response to Abiotic Stresses. Plants 2021, 10, 593. https://doi.orgdoi:10.3390/ plants10030593

Received: 14 February 2021

Accepted: 19 March 2021

Published: 22 March 2021

Publisher's Note: MDPI stays neutral with regard to jurisdictional claims in published maps and institutional affiliations.

Copyright: (c) 2021 by the authors. Licensee MDPI, Basel, Switzerland. This article is an open access article distributed under the terms and conditions of the Creative Commons Attribution (CC BY) license (https:// creativecommons.org/licenses/by/ $4.0 /)$.

\begin{abstract}
Zinc finger-homeodomain transcriptional factors (ZF-HDs), a kind of plant-specific transcription factor, play important roles in plant growth, development and various stress responses. In this study, the genome-wide analysis of the ZF-HD gene family was performed in wheat. A total of 37 TaZF-HD genes were identified in T. aestivum and classified into six groups. The results of a synteny analysis showed that gene replication events contributed to the expansion of the TaZF-HD gene family. The TaZF-HD paralogous gene pairs with similar chromosomal locations in different subgenomes had similar expression patterns. TaZF-HDs were highly induced under PEG (polyethylene glycol), $\mathrm{NaCl}$ and cold stress but not induced under heat stress. Gene ontology (GO) annotation and protein-protein interactions suggested that TaZF-HD proteins may participate in various biological processes of plants. These results increase our understanding of ZF-HD genes and provide robust candidate genes for future functional investigations aimed at crop improvement.
\end{abstract}

Keywords: ZF-HD genes; genome-wide identification; gene expression; wheat (Triticum aestivum L.)

\section{Introduction}

Transcription factors (TFs) have been confirmed to play central roles in the regulatory networks of plant growth, development and stress responses through binding to specific cis-elements [1,2]. For example, WRKY, NAC and GRF transcription factors are associated with seed development, leaf senescence, plant proliferation and expansion [3-5] whereas MYB, WRKY and NAC transcription factors can participate in the response of plants to biotic and abiotic stresses [6-8]. Zinc finger-homeodomain (ZF-HD) proteins are a kind of plant-specific transcription factor that plays vital roles in plant growth, development and various stress responses $[9,10]$.

ZF-HD proteins contain a homeodomain (HD) domain and a C2H2-type zinc finger (ZF) domain [11,12]. The HD domain is a well-characterized DNA binding domain (BD), which can be involved in the growth and development of plants by binding to DNA to regulate the expression levels of target genes [11,13]. Most homeodomain proteins have other domains that participate in protein-protein interaction and other functions [14]. The ZF domain widely exists in regulatory proteins and participates in DNA binding and protein-protein interactions [15]. ZF-HD proteins usually bind to specific DNA sequences with a core consensus of ATTA and form homodimers and heterodimers [9].

ZF-HD proteins were first identified in the $\mathrm{C} 4$ plant Flaveria as a potential regulator of the gene encoding C4 phosphoenolpyruvate carboxylase (PEPCase) [13]. Subsequently, ZF$H D$ genes were identified in several plant species such as Arabidopsis thaliana [11], tomato (Solanum lycopersicum) [16], cotton (Gossypium hirsutum) [17], Chinese cabbage [12] and Tartary buckwheat (Fagopyrum tataricum) [18]. In Arabidopsis, ZFHD10 recruits TANDEM 
ZINC-FINGER PLUS3 (TZP) to bind to light-regulated elements and regulate hypocotyl elongation [19]. ZHD1 is induced after drought, salinity and abscisic acid (ABA) treatments and specifically binds to the promoter region of EARLY RESPONSE TO DEHYDRATION STRESS1 (ERD1) [10]. In addition, the overexpression of NAC and ZHD1 enhances drought tolerance in Arabidopsis [10]. In soybeans, the expression of GmZF-HD1 and GmZF-HD2 are induced after pathogen inoculation and bind to the promoter region of the calmodulin subtype 4 gene (GmCaM4) [20]. Interestingly, mini zinc finger (MIF) genes identified in the ZF-HD gene family of Arabidopsis encode proteins with the ZF domain of ZF-HD proteins but without the HD domain [11]. Phylogenetic and sequence analyses of ZF-HD genes demonstrated that both ZFDs and MIFs are land plant-specific and they belong to two different groups of the ZF-HD gene family [11]. However, the origin and evolution of the ZHD and MIF genes remain unclear. MIFs might be derived from ZHDs by losing the HD domain; alternatively, ZHDs might have originated from MIFs by gaining the HD domain [11].

ZF-HD genes have been identified in several plant species; however, a genome-wide identification of $Z F-H D$ genes in wheat has not been performed. Fortunately, whole genome sequence data of the bread wheat "Chinese Spring" were published [21], making it possible to identify and analyze the ZF-HD gene family in wheat. Therefore, in this study, we performed a genome-wide analysis of ZF-HD genes in wheat to characterize their sequences, evolutionary relationships and expression patterns in different tissues and under various abiotic stress treatments.

\section{Results}

\subsection{Characteristics and Phylogenetic Analysis of TaZF-HDs in T. aestivum}

A total of 37 ZF-HD proteins were identified from a wheat genome based on a Hidden Markov Model (HMM) search of a ZF-HD dimerization region (PF04770) (Table S1). Based on chromosome locations and the phylogenetic relationship of TaZF-HDs, we renamed them from TaZHD1 to TaZHD28 and TaMIF1 to TaMIF9. The 37 predicted TaZF-HD genes encoded polypeptides of 93-465 amino acids in length with predicted molecular weights ranging from 9.84 (TaMIF6) to 50.6 (TaZHD9) $\mathrm{kDa}$ and the isoelectric points (pI) ranged from 6.17 (TaMIF1) to 9.89 (TaZHD3). The calculated grand average of hydropathy index (GRAVY) values of all TaZF-HDs were below zero $(-0.309$ to -1.14$)$, suggesting that they were hydrophilic. A subcellular localization prediction indicated that TaZF-HD proteins were all located in the nuclear. The phylogenetic tree was constructed with TaZF-HD, AtZF-HD and OsZF-HD proteins (Table S2). The results indicated that TaZF-HD proteins were divided into six groups (Figures 1 and $2 \mathrm{~A}$ ), which were consistent with previous phylogenetic analyses of plant ZF-HD proteins $[16,18]$. Group II and MIF were the largest with 10 and 9 members, respectively. Group I, III, IV and V included 7, 3, 3 and 3 members, respectively (Figure 1 and Table S1). 


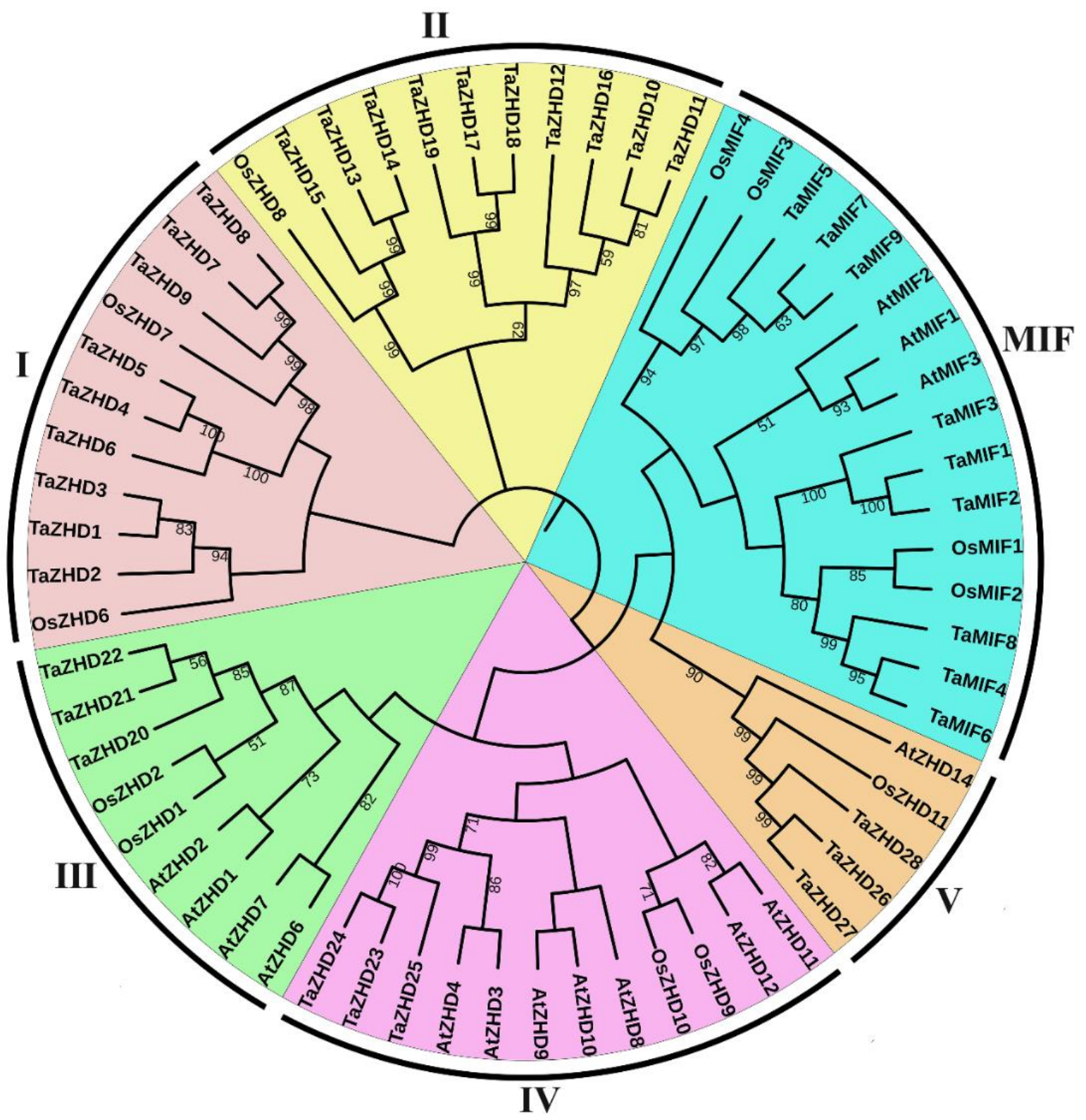

Figure 1. The neighbor-joining (NJ) phylogenetic tree of ZF-HD proteins. The tree was constructed with amino acid sequences of identified ZF-HD proteins from in A. thaliana (At), O. sativa (Os) and T. aestivum (Ta) and bootstrap values of 1000 replicates. Different groups of ZF-HD proteins are distinguished by different colors. 
A

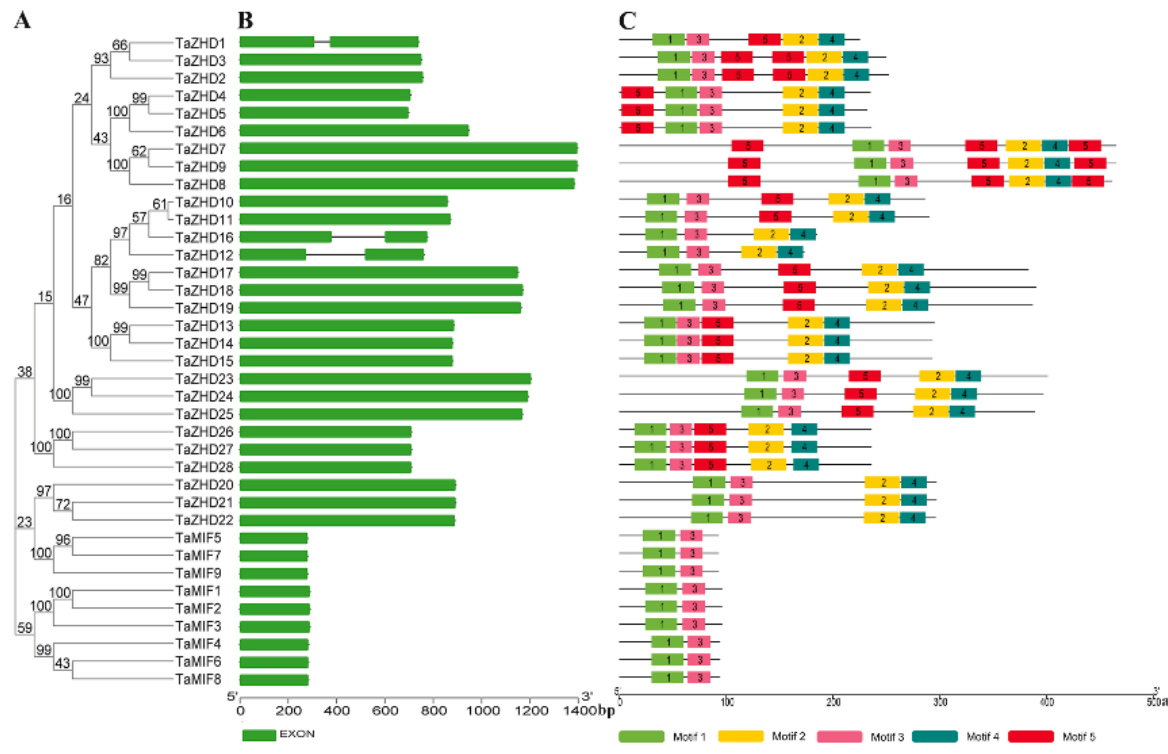

D

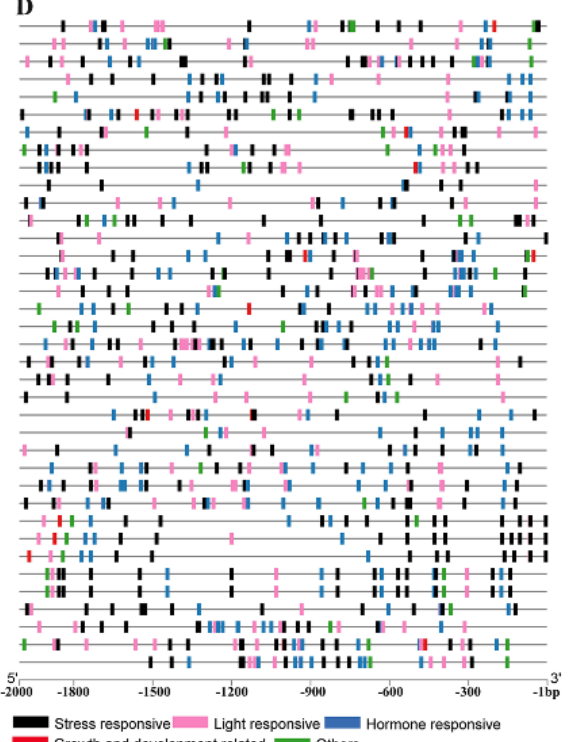

Figure 2. Phylogenetic relationships (A), exon-intron structures (B), conserved domains (C) and cis-elements analysis of TaZF-HD genes. (B) Green boxes and black lines indicated exons and introns, respectively. (C) Conserved domain compositions of TaZF-HD proteins in wheat. Motif 1 to motif 5 are shown in the different colors. (D) Hormone-responsive elements, stress-responsive elements, growth and development related elements, light-responsive elements and other elements with unknown functions are shown by different colors.

\subsection{Gene Structures, Conserved Motifs and Cis-Elements Analysis of TaZF-HDs}

To investigate the structural characteristics of the TaZF-HD genes, the exon-intron structures (Figure 2B) and conserved motifs (Figure 2C) of 37 TaZF-HD genes were analyzed. The exon-intron structures analysis indicated that most of the TaZF-HD genes did not include introns and only three TaZF-HDs (TaZHD1, 12 and 16) had one intron (Figure 2B and Table S1). To further investigate the diversity of TaZF-HD genes, the conserved motifs were analyzed using the MEME (Multiple Em for Motif Elicitation) online server (Figure 2C). Finally, five motifs were identified among of 37 TaZF-HD genes and named motif 1 to motif 5 (Figure 2C and Figure S1). All of the TaZF-HD proteins contained motif 1 and 3, which were typical ZF domains. Motif 2 and 4, the typical HD domains, were present in all TaZHDs (TaZHD1-TaZHD28) but were absent in TaMIFs (TaMIF1-TaMIF9) (Figures $2 \mathrm{C}$ and 3 ). These results verified the reliability of the identified members of the TaZF-HD gene family. The same group of TaZF-HDs had similar exon-intron structures and conserved motifs and TaZF-HDs with closer evolutionary relationships had more similar numbers and lengths of exons and conserved motifs (Figure 2).

The presence of multiple different cis-elements in the gene promoters might indicate that these genes perform different functions. To explore the cis-elements in the promoters of TaZF-HD genes, $2 \mathrm{~kb}$ of the upstream genomic sequence of each gene transcription start site (TSS) were extracted and then searched on the PlantCARE database to identify and count the cis-elements. These cis-elements could be divided into five categories: (i) hormone-responsive elements such as ABRE (ABA-responsive element), TGAelement (auxin-responsive element), CGTCA-motif (methyl jasmonate-responsive element), TGACG-motif (methyl jasmonate-responsive element), P-box (gibberellin-responsive element), TCA-element (salicylic acid-responsive element); (ii) stress-responsive elements such as DRE (dehydration-responsive element), LTRE (low temperature-responsive element), MBS (MYB binding site), MYC, STRE (stress-responsive element), W-box (WRKY transcription factor binding site), WRE3 (wounding-responsive element); (iii) growth and development related elements, CAT-box (cis-element related to meristem expression), circadian (cis-element related to circadian control) and RY-elements (cis-element related to seed-specific regulation); (iv) light-responsive elements such as ACE (AC-rich element), 
GA-motif, G-box, I-box; (v) other elements with unknown functions (Figure 2D). TaZF-HDs with closer evolutionary relationships showed a similar distribution of cis-elements in their promoters such as TaZHD20-TaZHD21 and TaMIF1-TaMIF2. These results indicated that the TaZF-HD genes may participate in the growth and development and respond to various stresses in wheat.

Motif 1

Motif 3

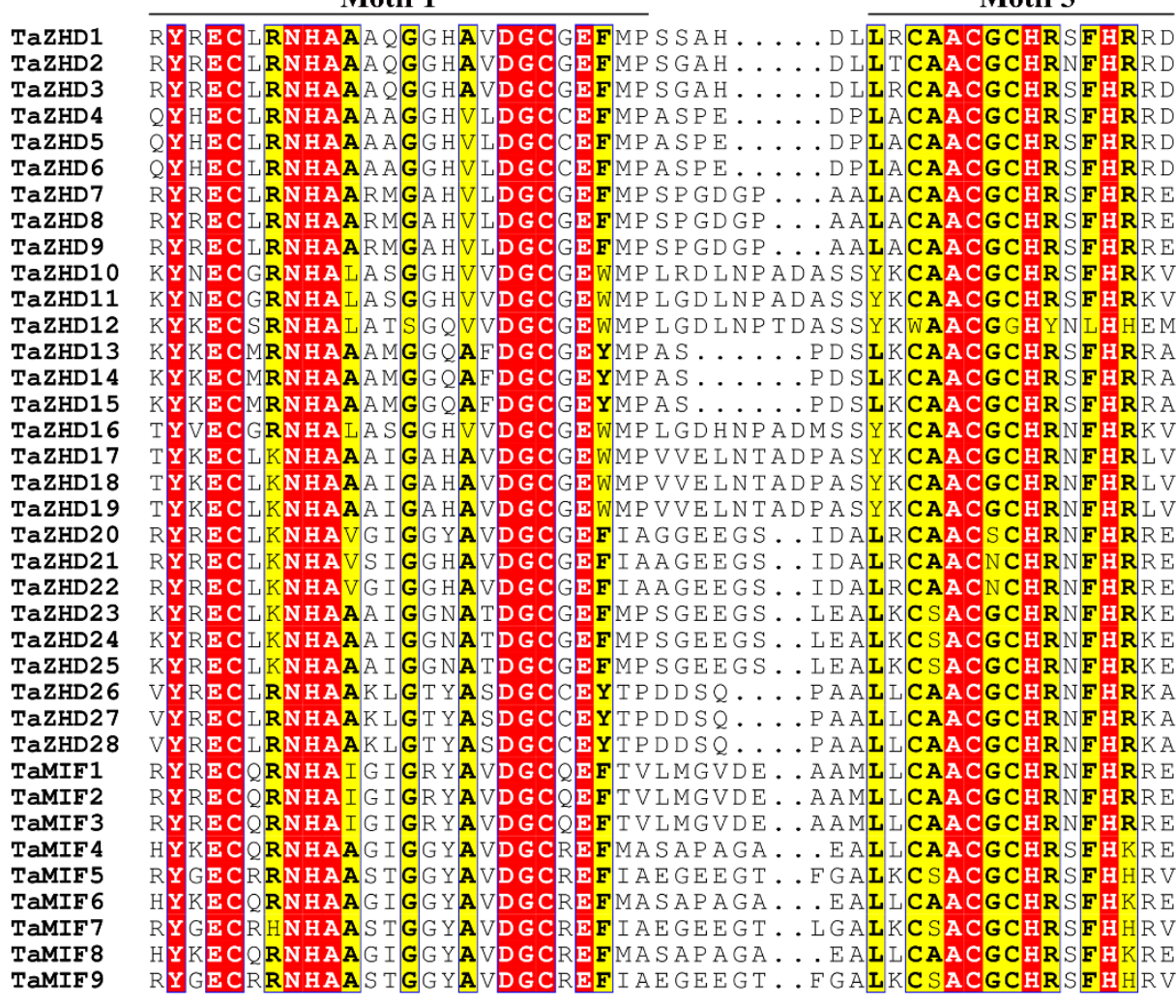

ZF domain

ZF domain

Motif 2

Motif 4

TazhD1

TazHD2

TazHD4

TazHD5

TazHD 6

TazHD7

TazHD8

TazHD10

TazHD10

TazHD11

TaZHD13

TazHD14

TaZHD15

TazHD16

TaZHD17

TazHD18

TazHD19

TazHD20

TazHD21

TazHD22

TazHD23

TazHD24

TazHD25

TazHD2 6

TaZHD27

TaZHD28

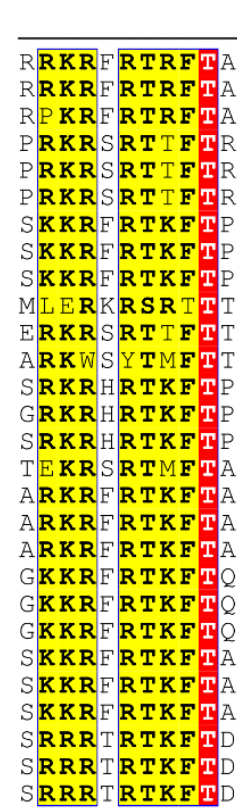

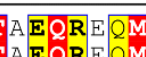

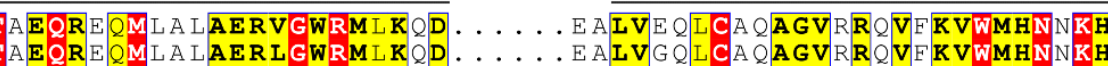

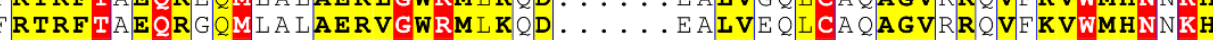

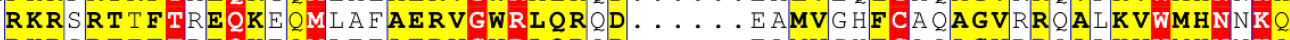

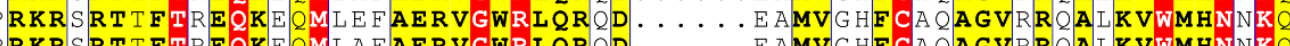

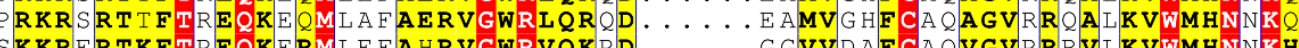

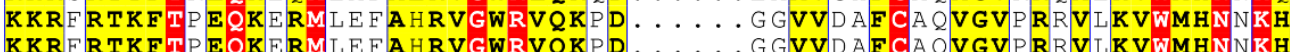

KKRFRTKFTP EQKERMLEFAHRVGWRVQKP D . . . . G GVVDAF CA QVGVPRRVLKVWMHNNKH

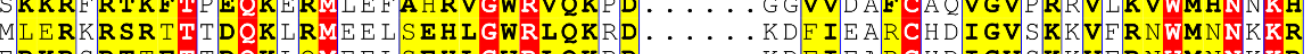

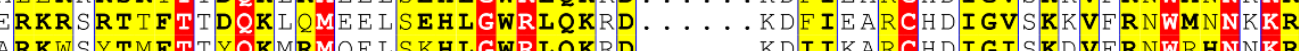

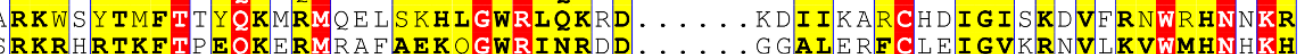

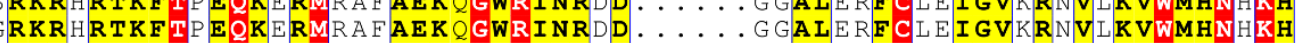

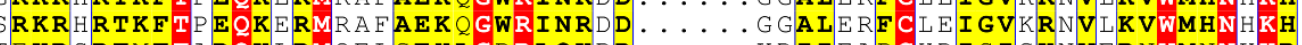

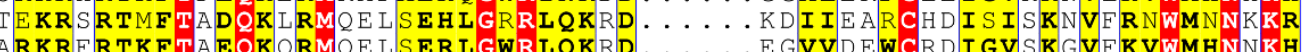

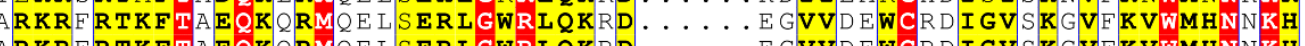

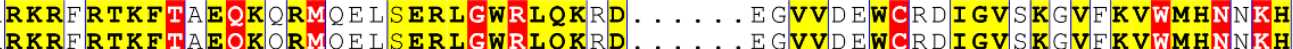

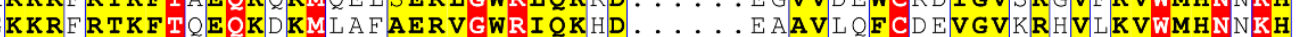

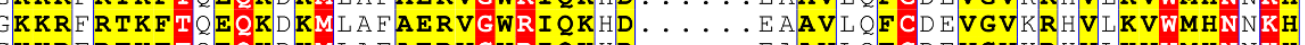

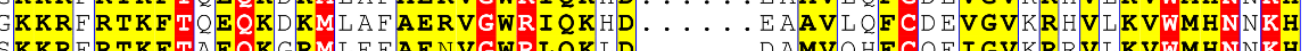

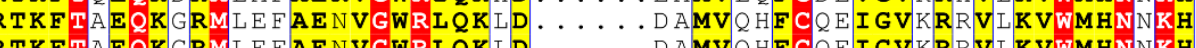

I
I

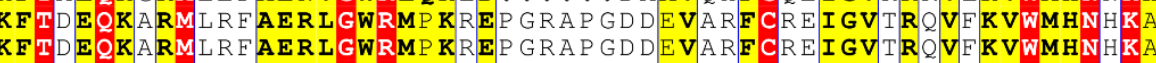

RMLRF AERLGWRMP KREP GRAP GD DEVARECREIGVTRQVF K
HD domain
HD domain

Figure 3. Multiple sequence alignment of the conserved domains of the members of the TaZF-HD gene family in wheat. Motifs 1 and 3 were ZF domains and motifs 2 and 4 were HD domains. 


\subsection{Chromosomal Location, Synteny and Ka/Ks Analysis of TaZF-HDs}

The chromosomal locations of the identified TaZF-HD genes in wheat were mapped to the corresponding chromosomes by the MapChart and circos tools (Figure 4 and Figure S2). TaZF-HD genes were distributed in chromosome 1, 2, 3, 4, 5, 6 and Un. Chromosome 5, which was the chromosome with the most TaZF-HD genes, contained twelve TaZF-HD genes and chromosome 7 had no TaZF-HD genes. TaZF-HD genes were approximately evenly and similarly distributed in the A, B and D subgenomes.

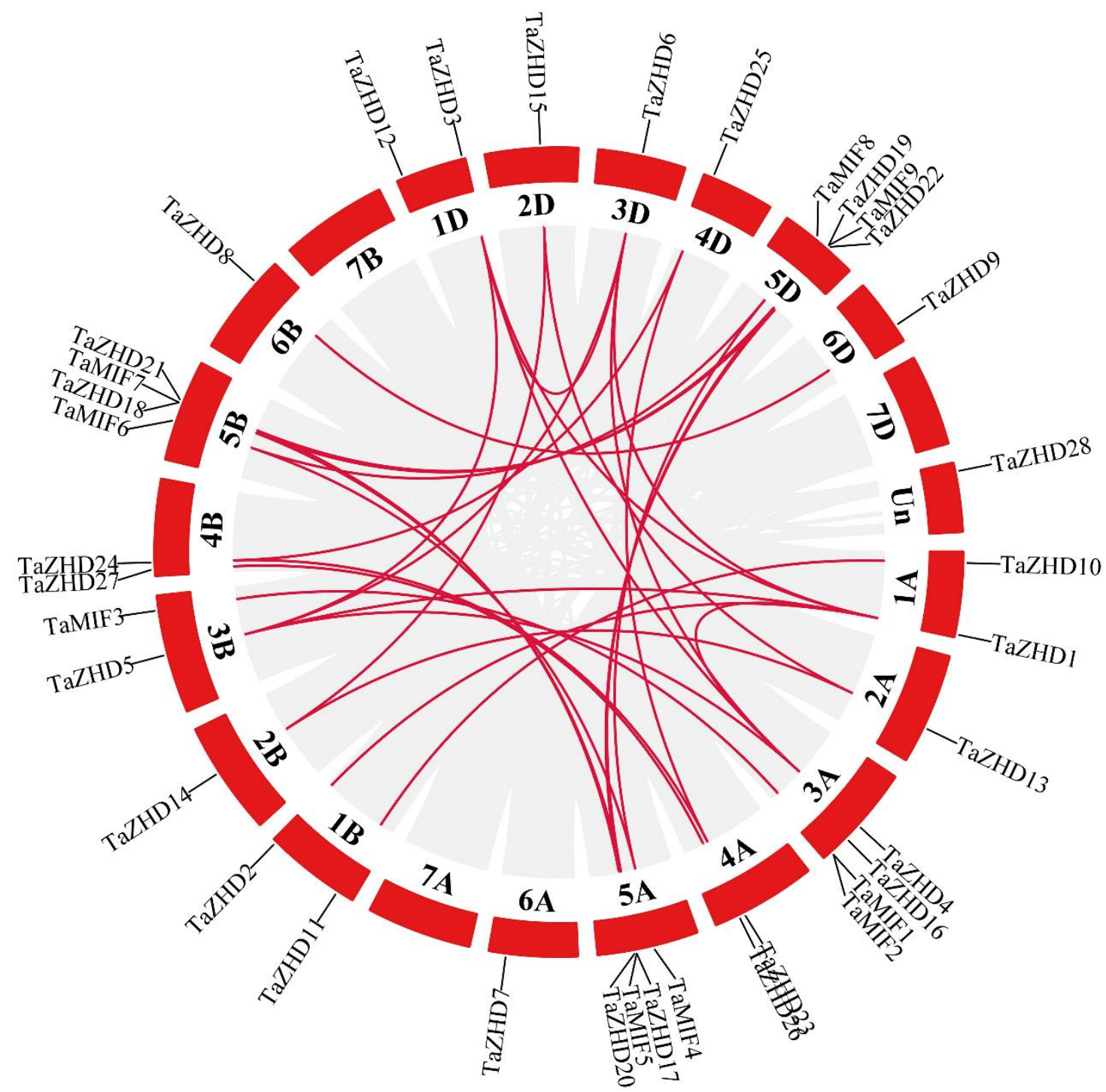

Figure 4. Chromosomal localizations and syntenic relationships among TaZF-HD genes in T. aestivum. Gray lines in the background indicate the collinear blocks among T. aestivum and red lines highlight the syntenic TaZF-HD gene pairs.

Synteny analysis suggested that 36 paralogous gene pairs were found among $37 \mathrm{TaZF}$ $H D$ genes in wheat with the most gene duplication events on chromosome $1 \mathrm{~A}$ and only one on chromosome 6B and 6D, respectively (Figure 4 and Table S3). Moreover, 32 TaZF$H D$ s had undergone WGD (whole genome duplications) or segmental duplication events whereas only one gene (TaMIF2) was a tandem replication. These results showed that most TaZF-HD genes might be produced by fragment replication events and these replication events played a vital function in the evolution of TaZF-HD genes. To investigate the selective pressure on the duplicated TaZF-HD genes, the non-synonymous (Ka) and synonymous substitution (Ks) values were calculated for the 36 paralogous gene pairs (Table S3). The value of $\mathrm{Ka} / \mathrm{Ks}=1$ meant that genes experienced a neutral selection, $\mathrm{Ka} / \mathrm{Ks}>1$ indicated a 
positive selection and $\mathrm{Ka} / \mathrm{Ks}<1$ suggested a purifying/negative selection [22]. The values of $\mathrm{Ka} / \mathrm{Ks}$ from all 36 paralogous gene pairs were less than 1, which indicated that these TaZF-HD genes had undergone a strong purifying/negative selection pressure with little changed after duplication.

To further investigate the synteny relationships of $Z H-H D$ genes between the T. aestivum (AABBDD, hexaploid) with T. urartu (AA, diploid), Ae. tauschii (DD, diploid), B. distachyon (diploid) and O. sativa (diploid), a Multiple Collinearity Scan toolkit (MCScanX) was used to identify the orthologous genes among these released plant genomes (Figure 5 and Table S4). We identified 18, 35, 41 and 39 orthologous gene pairs between TaZF-HDs with other $Z H-H D$ genes in $T$. urartu, Ae. tauschii, B. distachyon and $O$. sativa, respectively. The results showed that $18,29,28$ and $25 \mathrm{TaZF}-H D$ s genes were collinear with $Z H-H D$ genes in T. urartu, Ae. tauschii, B. distachyon and O. sativa, respectively. A few TaZF-HD genes had at least two pairs of orthologous genes such as TaZHD1, TaZHD2, TaZHD5 and TaMIF8, which might have played a vital function in the evolution of ZF-HD genes. These results indicated that $\mathrm{TaZH}-\mathrm{HD}$ genes in wheat might be derived from orthologous genes in other plant species.

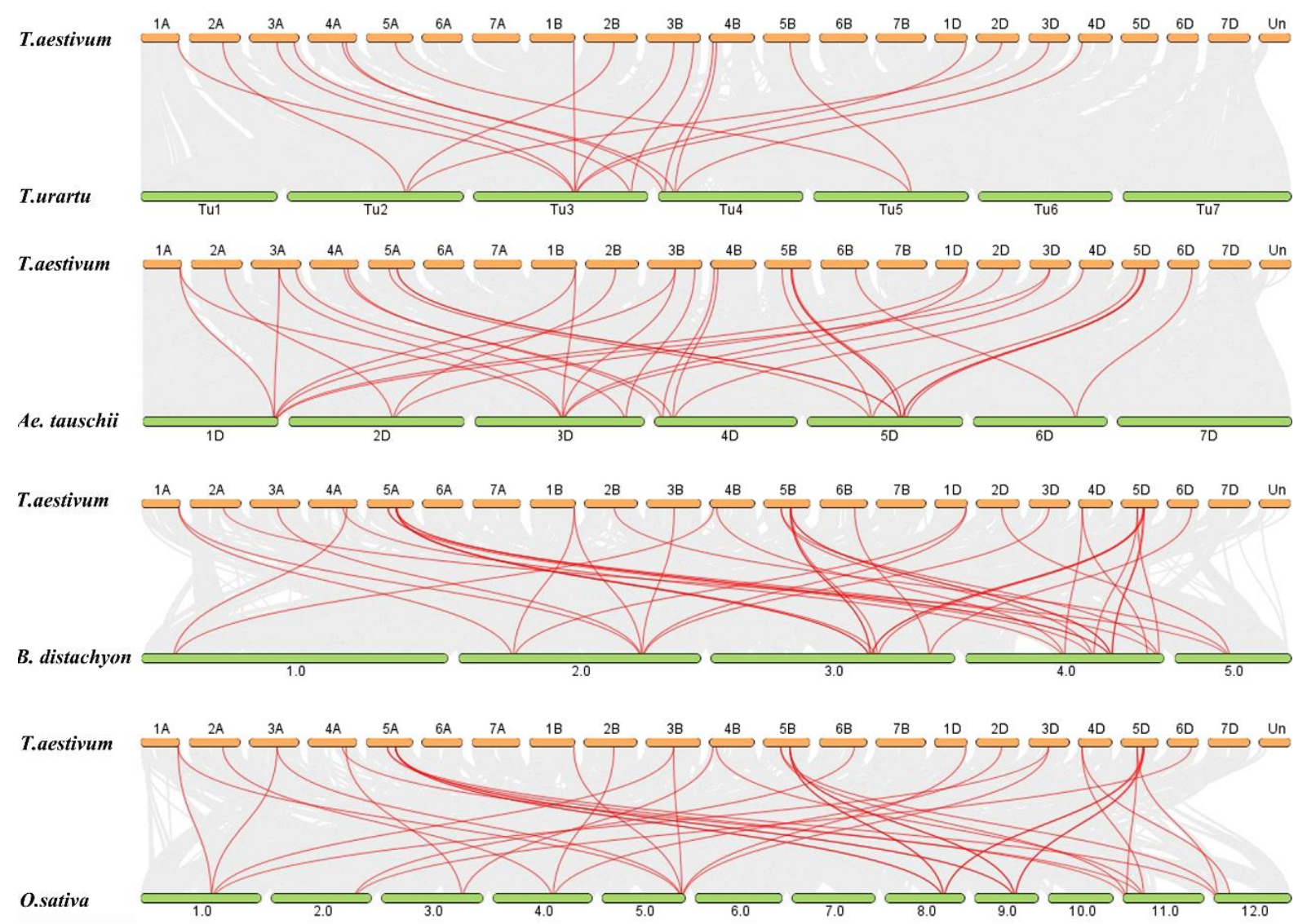

Figure 5. Syntenic relationships between TaZF-HD genes in T. aestivum with other ZF-HD genes in four representative plant species. Gray lines in the background indicate the collinear blocks within T. aestivum and other plant genomes and red lines highlight the syntenic ZF-HD gene pairs.

\subsection{Expression Patterns of TaZF-HD Genes in Different Tissues}

To investigate the tissue-specific expression patterns of the TaZF-HD genes in wheat, the RNA-seq data in wheat seedling, vegetative and reproductive stages were obtained from the expVIP website (Figure 6 and Table S5). Most TaZF-HD genes exhibited tissuespecific expression patterns and could be detected in the leaves, spikes and grains but not expressed in the roots. Many paralogous gene pairs with similar chromosomal locations in different subgenomes had more similar expression patterns; for example, TaZHD1, 
TaZHD2 and TaZHD3 were mainly expressed in grains at 30 days post-anthesis (dpa); TaMIF1, TaMIF2 and TaMIF3 were highly expressed in grains at 2 dpa and in a whole endosperm at $10 \mathrm{dpa}$. Fewer TaZF-HD gene pairs were expressed in most tissues; for example, TaMIF4, TaMIF6 and TaMIF8 exhibited higher expression levels in almost all tissues except in grains at 30 days post-anthesis (dpa); TaZHD4, TaZHD5 and TaZHD6 were also highly expressed in most tissues. However, a few paralogous gene pairs had different expression patterns in various tissues, e.g., TaZHD10 had higher expression levels in the whole endosperm, aleurone layer and starchy endosperm and TaZHD11 was almost not expressed (Figures 4 and 6).

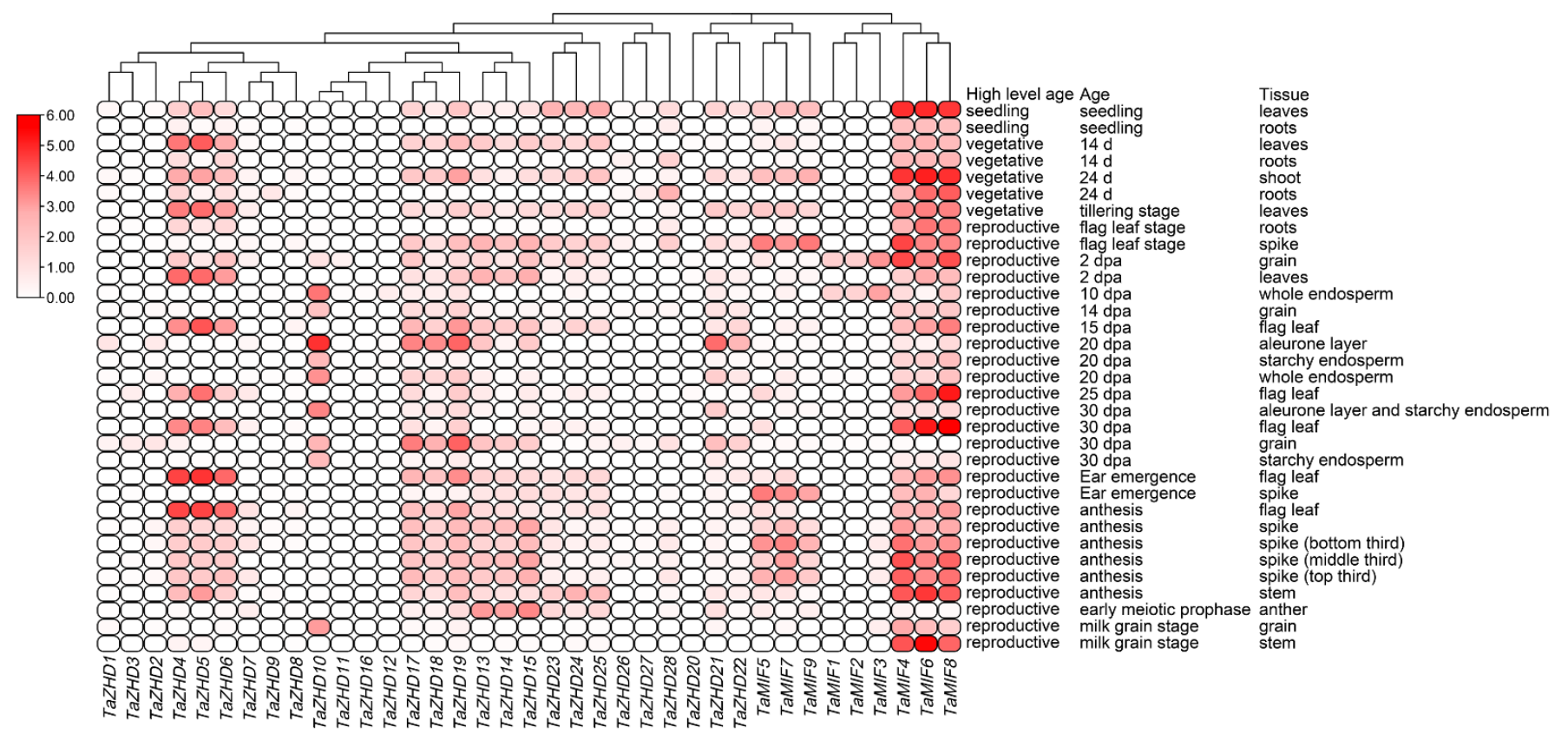

Figure 6. Tissue-specific expression patterns of the TaZF-HD genes in various wheat tissues. The $\log _{2}$ of TPM (transcripts per million) values were calculated by RNA-seq data to show the expression levels of the TaZF-HD genes in wheat.

We also determined the expression profiles of ten TaZF-HD genes belonging to I (TaZHD4), II (TaZHD10, TaZHD13 and TaZHD14), III (TaZHD20 and TaZHD21), IV (TaZHD24), V (TaZHD28) and MIF (TaMIF4 and TaMIF6) groups in the roots, stems and leaves of wheat seedlings by real-time PCR (Figure 7). The results indicated that nine of these ten TaZF-HD genes (TaZHD10, TaZHD13, TaZHD14, TaZHD20, TaZHD21, TaZHD24, TaZHD28, TaMIF4 and TaMIF6) had the highest expression levels in the leaves followed by the roots. The expression level of TaZHD4 was the highest in the roots followed by the leaves. All of these ten TaZF-HD genes had the lowest expression levels in the stems. These results suggested that these ten TaZF-HD might play important roles in the development process of the leaves.

\subsection{Expression Patterns of TaZF-HD Genes under Abiotic Stresses}

To further investigate the function of TaZF-HD genes, real-time PCR was used to detect the expression profiles of ten TaZF-HD genes (TaZHD4, TaZHD10, TaZHD13, TaZHD14, TaZHD20, TaZHD21, TaZHD24, TaZHD28, TaMIF4 and TaMIF6) under PEG (polyethylene glycol), $\mathrm{NaCl}$ and heat and cold stresses in wheat leaves at the seedling stage (Figure 8). In PEG treatment conditions, all TaZF-HD genes were initially up-regulated and then down-regulated and reached the highest expression level at $12 \mathrm{~h}$ (2.2- to 25.9-fold vs. the control). Under $\mathrm{NaCl}$ stress, the expression of six TaZF-HD genes (TaZHD4, TaZHD10, TaZHD13, TaZHD14, TaZHD20 and TaZHD28) peaked at $36 \mathrm{~h}$ after treatment (1.7- to 3.8-fold vs. the control). TaZHD21, TaZHD24, TaMIF4 and TaMIF6 were down-regulated after salt stress treatment compared with the control. Under heat stress, TaZHD4 and TaZHD28 
were up-regulated and other TaZF-HD genes were down-regulated compared with the control. Under cold stress, eight TaZF-HD genes (TaZHD4, TaZHD10, TaZHD13, TaZHD14, TaZHD20, TaZHD21, TaZHD24 and TaZHD28) were the most highly expressed at $12 \mathrm{~h}$ or $24 \mathrm{~h}$ (1.3- to 16.7-fold) compared with the control. TaZHD4 and TaZHD28 were up-regulated under all abiotic stresses. Paralogous genes also had similar expression patterns under abiotic stress, e.g., TaMIF4 and TaMIF6 had almost consistent expression patterns under PEG, $\mathrm{NaCl}$, heat or cold stress.

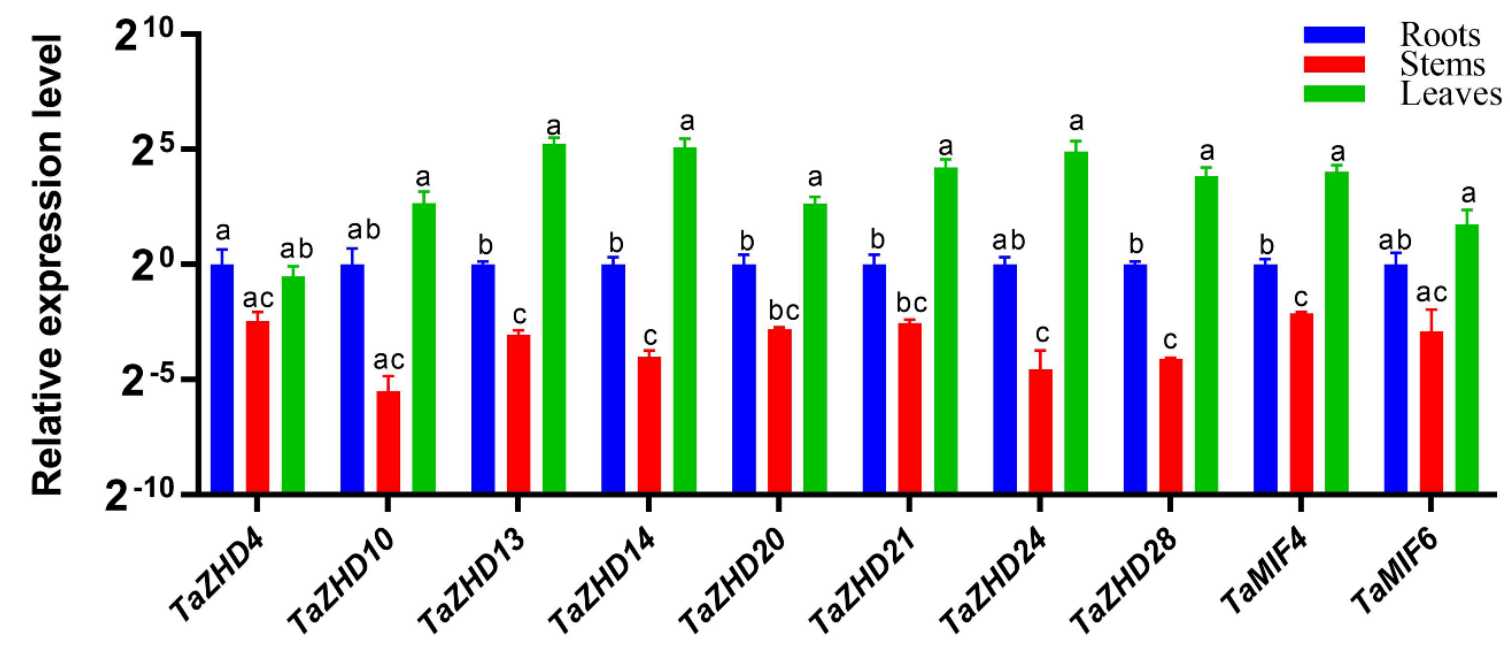

Figure 7. Expression levels of TaZF-HD genes in the roots, stems and leaves of wheat seedlings. The expression level of the wheat actin gene was used as the internal control to standardize the RNA samples for each reaction. The values are the mean \pm SE from three samples. Statistical significance was determined by a student's $t$-test and different lowercase letters indicate significant differences $(p<0.05)$ for each parameter.
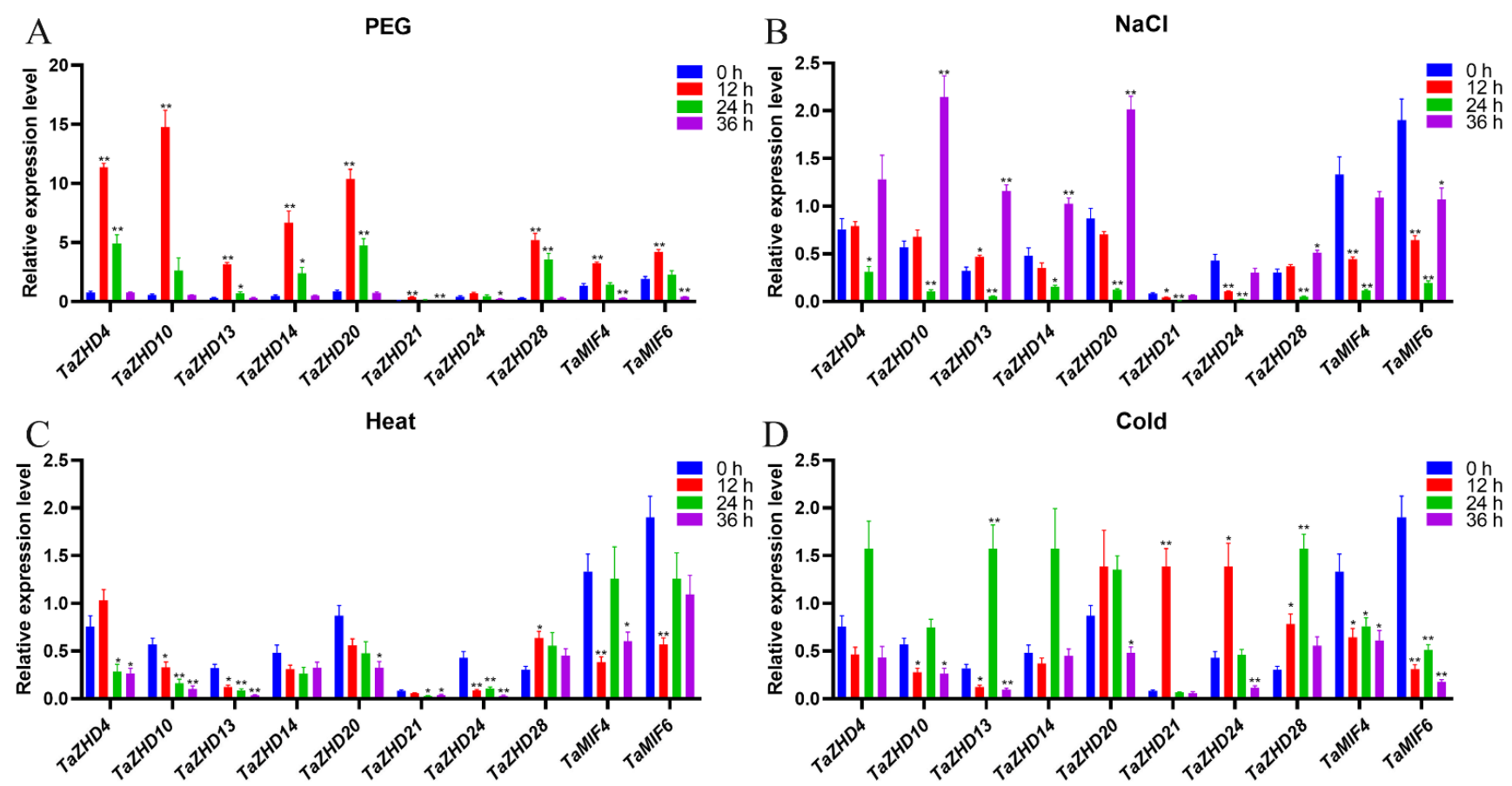

Figure 8. Expression patterns of TaZF-HD genes in response to PEG (polyethylene glycol) (A), $\mathrm{NaCl}(\mathbf{B}), \mathrm{heat}(\mathbf{C})$ and cold (D) treatments determined by real-time PCR. The expression level of the wheat actin gene was used as the internal control to standardize the RNA samples for each reaction. The values are the mean $\pm \mathrm{SE}$ from three samples and significant differences were indicated as $\left(^{*}\right) p<0.05$ and $\left(^{* *}\right) p<0.01$. 


\subsection{GO Annotation Analysis and Protein-Protein Interactions of TaZF-HDs}

Thirty-two of the thirty-seven TaZF-HD proteins could be annotated by gene ontology (GO), which were contributed to understand the function from molecular levels. The 32 TaZF-HD proteins were assigned with $20 \mathrm{GO}$ terms belonging to the cellular component, molecular function and biological process (Figure 9A). Among these 32 TaZF-HD proteins, 30 TaZF-HDs were located in the nucleus (GO:0005634). Under the molecular function category, all of the TaZF-HDs were involved in nucleic acid binding (GO:0003676) and 25 TaZF-HDs could participate in DNA binding transcription factor activity (GO:0003700). Under the biological process, 25 TaZF-HDs were involved in the cellular metabolic process (GO:0044237) and the cellular nitrogen compound metabolic process (GO:0034641).

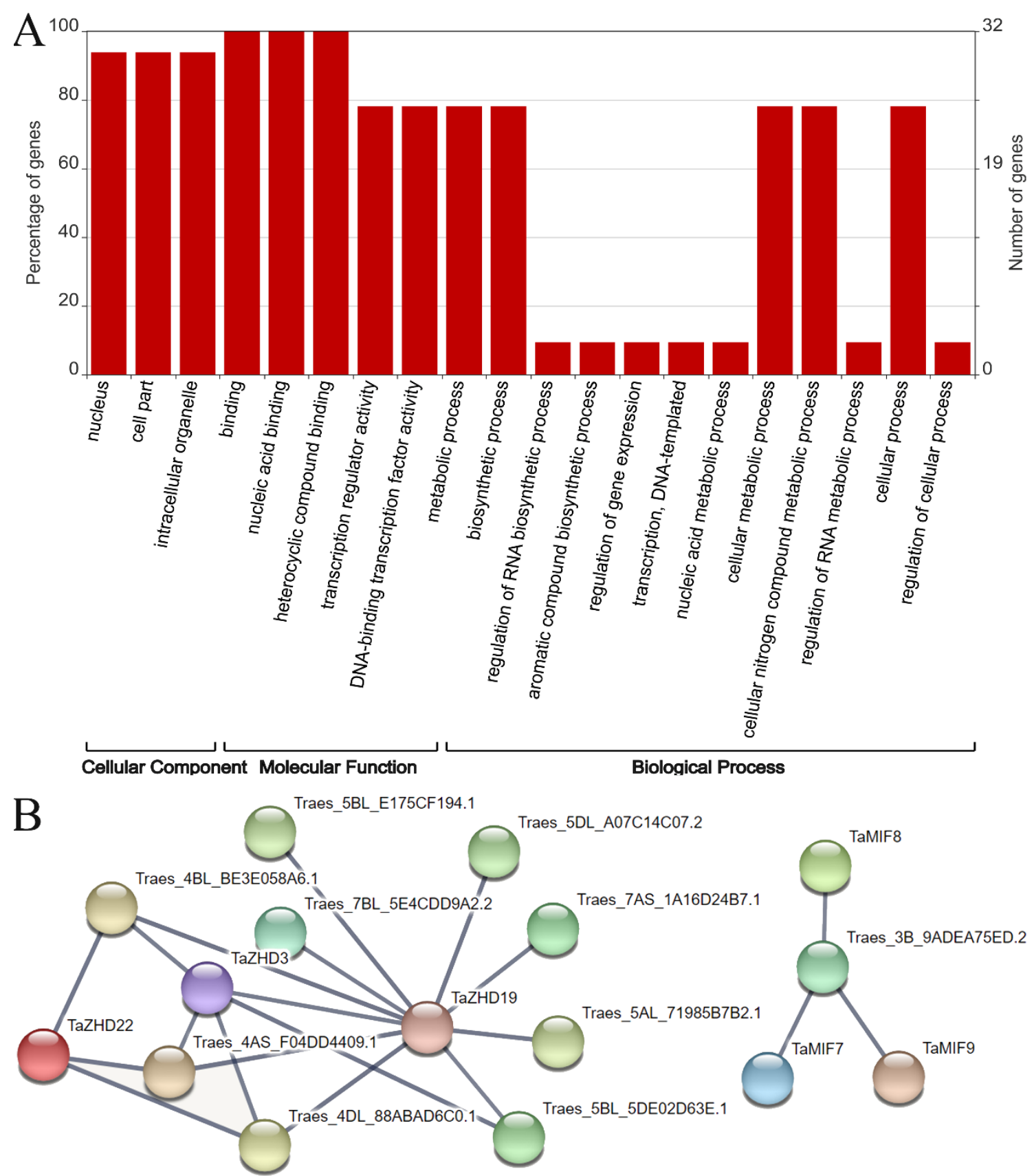

Figure 9. Gene ontology (GO) annotation (A) and protein-protein interactions (B) analysis of TaZF-HD proteins. 
To investigate protein-protein interactions between TaZF-HDs and other wheat proteins, a network was constructed using the STRING database (Figure 9B and Table S6). According to the predicted results, we identified six TaZH-HDs interacting with 10 other wheat proteins. TaZHD19 could interact with TaZHD3 and nine other wheat proteins, which were homeodomain-leucine zipper transcription factors (Traes_4AS_F04DD4409.1, Traes_4BL_BE3E058A6.1, Traes_4DL_88ABAD6C0.1, Traes_5BL_5DE02D63E.1), a mediator of RNA polymerase II transcription subunit 25 (Traes_5AL_71985B7B2.1, Traes_5BL_E175CF194.1, Traes_5DL_A07C14C07.2), cytochrome P450 (Traes_7AS_1A16D24B7.1) and macrophage migration inhibitory factor (Traes_7BL_5E4CDD9A2.2), suggesting that TaZHD19 played a pivotal role in the regulation of protein networks. TaZHD3 and TaZHD22 also interacted with three and four kinds of homeodomain-leucine zipper transcription factors, respectively. TaMIF7, TaMIF8 and TaMIF9 could all interact with the GATA-type zinc finger protein (Traes_3B_9ADEA75ED.2). These results provided a valuable foundation for future functional investigations of TaZF-HD genes.

\section{Discussion}

\subsection{Evolution and Expansion of the TaZF-HD Gene Family in Wheat}

The members of the ZF-HD gene family have been reported in many plants but they have not been genome-wide identified in wheat. Previous studies indicated that ZF-HD genes only existed in land plants and expanded during angiosperm evolution [11,12]. To further investigate the synteny relationships of $\mathrm{ZH}-\mathrm{HD}$ genes between the wheat and other plant species, we identified 18, 35, 41 and 39 orthologous gene pairs between TaZF-HDs with other ZH-HD genes in T. urartu, Ae. tauschii, B. distachyon and O. sativa, respectively (Figure 5 and Table S4). Moreover, T. urartu (AA, diploid) and Ae. tauschii (DD, diploid) were the source of wheat (AABBDD, hexaploid) A and D subgenomes. The synteny analysis indicated that six orthologous gene pairs between T. urartu with a wheat A subgenome were located on the same chromosomes with one on $2 \mathrm{~A}$, two on $3 \mathrm{~A}$, two on $4 \mathrm{~A}$ and one on 5A. Meanwhile, nine orthologous gene pairs between Ae. tauschii with a wheat D subgenome were located on the same chromosomes with one on $1 \mathrm{D}$, one on $2 \mathrm{D}$, one on 3D, one on 4D, four on 5D and one on 6D (Figure 5 and Table S4). These TaZF-HD genes might be derived from orthologous genes in T. urartu and Ae. tauschii with the occurrence of natural hybridization events. Furthermore, more orthologous gene pairs were identified between wheat with $B$. distachyon and O. sativa, which suggested that TaZF-HDs and other $\mathrm{ZH}-\mathrm{HD}$ genes in $B$. distachyon and $O$. sativa might be derived from a common ancestor with a long-term evolutionary process.

The gene duplication events including tandem, segmental and whole genome duplications are primary driving forces of the expansion of the gene family in plant genome evolution $[23,24]$. In this study, we identified $37 \mathrm{TaZF-HD}$ genes in wheat including 28 TaZF-HD genes and nine TaMIF genes (Figure 1 and Table S1). The number of TaZF-HD genes was relatively higher than that identified in Arabidopsis (17) [11], tomato (22) [16], Chinese cabbage (31) [12] and Tartary buckwheat (20) [18] and the same as that in cotton (37) [17], suggesting that genome duplication events might have contributed to the expansion of TaZF-HD genes in wheat. Thirty-six paralogous gene pairs were identified in wheat, which all had undergone WGD or segmental duplication events and a strong purifying selection pressure (Figure 4 and Table S3). In conclusion, WGD or segmental duplications played vital roles in the evolution and expansion of the TaZF-HD genes.

\subsection{Expression and Function Analysis of TaZF-HD Genes in Wheat}

$Z F-H D$ genes participate in various biological processes and play crucial roles in plant growth, development and stress responses $[9,10]$. The tissue-specific expression profiles usually reveal their corresponding biological functions [25,26]. In Arabidopsis, most $Z F-H D$ genes are expressed in floral tissues, indicating a likely regulatory role during floral development [11]. TaZFHD1, described as TaZHD19 in this study, was differentially expressed during spike development with a preferential expression during "half emerged", 
"completely emerged" and "half anthesis" stages [27]. In this study, TaZHD19 also had a higher expression level in the spike during the reproductive stage (Figure 6). These results indicated that TaZFD19 might be involved in wheat inflorescence development and/or pollination [27]. Most TaZF-HD genes exhibited relatively higher expression levels in the leaves, spikes and grains and lower expression levels in the roots (Figures 6 and 7), which indicated TaZF-HDs might play an important function in their growth and development. Paralogous gene pairs with similar chromosomal locations in different subgenomes had more similar expression patterns, e.g., TaZHD1, TaZHD2 and TaZHD3, indicating that they might have redundant functions in regulating plant growth and development (Figures 4 and 6).

Previous studies have shown that $Z F-H D$ genes were induced by drought and high salinity and an overexpression of NAC and ZHD1 activated the expression of ERD1 and enhanced drought tolerance in Arabidopsis [10]. Most TaZF-HD genes were also highly expressed after PEG, $\mathrm{NaCl}$ and cold treatments but not expressed under heat stress (Figure 8). Similarly, $V v Z H D$ genes were up-regulated in response to dehydration or high salinity stresses in grapes [28]. SlZHD18 was obviously induced by drought, $\mathrm{NaCl}$ and cold treatments but not by heat stress [16].

According to previous studies, ZF-HD proteins can form homodimers and heterodimers or interact with other proteins to regulate plant growth, development and stress responses [9,10]. In Arabidopsis, ZFHD10 interacted with TZP protein to regulate hypocotyl elongation [19]. L1ZHFD4 interacted with NAC transcription factor LINAC2 to regulate the stress tolerance of the tiger lily [29]. The predicted results of the STRING database showed that TaZHD3 might interact with TaZHD19 (Figure 9B and Table S6); however, TaZHD3 was not co-expressed with TaZHD19 under abiotic stress (Figure S3) suggesting that TaZHD3 and TaZHD19 might perform other unknown functions in wheat growth and development via interacting with each other. TaMIF7, 8 and 9 had higher expression levels in the spike and might interact with Traes_3B_9ADEA75ED.2 (GATAtype zinc finger protein) suggesting that they might be involved in regulating wheat spike development via interacting with the GATA-type zinc finger protein (Figures 6 and 8B). TaZH-HD genes also interacted with the homeodomain-leucine zipper transcription factor and cytochrome $\mathrm{P} 450$, etc. These results provide valuable foundations for future functional investigations of TaZF-HD genes and breeding new wheat varieties.

\section{Materials and Methods}

\subsection{Identification of the ZF-HD Family Genes}

The genome sequence of T. aestivum was downloaded from the EnsemblPlant database (http://plants.ensembl.org/index.html). The Hidden Markov Model (HMM) profiles (http:/ / pfam.xfam.org) of the ZF-HD dimerization region (PF04770) were obtained from the Pfam database (http:// pfam.xfam.org) and were used to HMM search against the local genome database of T. aestivum using TBtools [30]. All of the identified TaZF-HD candidates were submitted to the Pfam database (http:/ / www.ebi.ac.uk/Tools/hmmer/) to confirm the ZF-HD protein conserved domains. We then retrieved 37 TaZF-HD genes. The physiological and biochemical parameters of the TaZF-HD proteins were analyzed by the ProtParam tool (http:/ /web.expasy.org/protparam/) and the subcellular localization of the TaZF-HD proteins was predicted using the Plant-mPLoc (http:/ /www.csbio.sjtu. edu.cn/bioinf/plant-multi/) and ProtComp 9.0 tools (http:/ /www.softberry.com/berry. phtml?topic=protcomppl\&group=programs\&subgroup=proloc).

\subsection{Phylogenetic Relationships, Gene Structures, Conserved Motifs and Cis-Elements Analysis}

The phylogenetic tree was constructed by the neighbor-joining (NJ) method with 1000 bootstrap replicates in the MAFFT and ITOL online service [31,32]. The exon-intron structures were identified using the Gene Structure Display Server (GSDS) (http:/ / gsds.cbi. pku.edu.cn/) by comparing CDS and genomic DNA sequences [33]. The conserved motifs were annotated using the MEME online server (http://meme-suite.org/index.html). The 
promoter sequences, which were $2000 \mathrm{bp}$ upstream of the transcription start site (TSS) of the TaZF-HD genes, were acquired from the T. aestivum database and the cis-elements in the promoters were analyzed in the PlantCARE database [34].

\subsection{Chromosomal Location, Synteny and Ka/Ks Analysis}

The chromosomal locations of each TaZF-HD gene were obtained according to genome annotation data and then marked on the chromosomes using the MapChart and cir$\cos [35,36]$. Multiple collinear scanning toolkits (MCScanX) were used to detect the gene replication events [37]. TBtools was used to determine the Ka and Ks of the syntenic gene pair with the Nei-Gojobori (NG) method [30].

\subsection{Gene Ontology Annotation and Protein-Protein Interactions Analysis}

GO annotation of TaZF-HD proteins was analyzed using the OmicsBox tool (https: / / www.biobam.com/) and displayed by the WEGO2.0 website (https:/ / wego.genomics. $\mathrm{cn} /$ ) [38]. Protein-protein interactions (PPIs) were predicted using the STRING database (https://string-db.org/). The combined score $>0.8$ in the STRING database was used to confirm the interaction network.

\subsection{Gene Expression Analysis}

To analyze the expression patterns of TaZF-HD genes in different tissues and stress conditions, the expression data were obtained from expVIP (http: / / www.wheat-expression. $\mathrm{com} /$ ) [39]. The TPM (transcripts per million) values of the TaZF-HD genes are presented in Table S5. The heatmap was drawn by TBtools [30].

\subsection{Plant Materials and Treatments}

Wheat seeds of "Chinese Spring" were germinated on moist filter paper at $25 / 18^{\circ} \mathrm{C}$ (day/night) with a photoperiod of $16 \mathrm{~h} /$ day. Wheat seedlings were grown in a hydroponic culture for two weeks and root, stem and leaf tissues of the wheat seedlings were collected. For the abiotic stress treatment, seedlings were exposed to 20\% PEG $6000(w / v)$, high salinity $(300 \mathrm{mM} \mathrm{NaCl})$, high temperature $\left(42^{\circ} \mathrm{C}\right)$ and cold $\left(4^{\circ} \mathrm{C}\right)$ conditions as described previously [40]. In each treatment, the leaf tissues were collected every $12 \mathrm{~h}$ for $36 \mathrm{~h}$. All samples were frozen in liquid nitrogen and stored at $-80^{\circ} \mathrm{C}$.

\subsection{RNA Isolation and Real-Time PCR Analysis}

An EasyPure Plant RNA Kit (TransGen) was used to isolate total RNA from each frozen sample and the first-strand cDNA was synthesized from total RNA $(1 \mu \mathrm{g})$ by using EasyScript One-Step gDNA Removal and cDNA Synthesis SuperMix (TransGen) according to the manufacturer's instructions. The sequence was amplified using gene-specific primers (Table S7) with TransTaq-T DNA Polymerase (TransGen) and the actin gene was used as an internal control. The real-time PCR cycling parameters were $94{ }^{\circ} \mathrm{C}$ for $30 \mathrm{~s}$ followed by 45 cycles at $94{ }^{\circ} \mathrm{C}$ for $5 \mathrm{~s}$ and $55^{\circ} \mathrm{C}$ for $30 \mathrm{~s}$ with a melting curve analysis from $60{ }^{\circ} \mathrm{C}$ to $90{ }^{\circ} \mathrm{C}$ at a rate of $0.5^{\circ} \mathrm{C} / 5 \mathrm{~s}$. All reactions were performed in triplicate to ensure the reproducibility of the results.

Supplementary Materials: The following are available online at https://www.mdpi.com/2223 -7747/10/3/593/s1, Figure S1: Conserved domains of TaZF-HD proteins in wheat. Figure S2: Chromosomal localizations of TaZF-HD genes in T. aestivum. Figure S3: The expression levels of TaZHD3 and TaZHD19 under drought, PEG, heat and cold stress. The $\log _{2}$ of the TPM values were calculated by RNA-seq data to show the expression levels of the TaZHD3 and TaZHD19 genes under drought, PEG, heat and cold stress in wheat. Table S1: The characteristics of ZF-HD genes in wheat. Table S2: ZF-HD genes used in the phylogenetic tree construction. Table S3. Paralogous ZH-HD gene pairs among T. aestivum. Table S4: Orthologous relationships between TaZF-HD genes in T. aestivum with other ZF-HD genes in T. urartu, Ae. tauschii, B. distachyon and O. sativa. Table S5: The expression levels of TaZF-HD genes in different tissues of wheat. Table S6: The protein-protein 
interaction network between TaZF-HDs and other proteins in wheat. Table S7: Real-time PCR primers of TaZF-HD genes.

Author Contributions: Conceptualization, H.L. and Y.Y.; methodology, H.L.; software, H.L.; validation, H.L. and Y.Y.; investigation, H.L.; resources, L.Z.; data curation, H.L.; writing-original draft preparation, H.L., Y.Y. and L.Z.; writing-review and editing, H.L.; funding acquisition, L.Z. All authors have read and agreed to the published version of the manuscript.

Funding: This research was funded by National Natural Science Foundation of China, grant number 31671608 .

Data Availability Statement: The public RNA-seq data were obtained from expVIP (http:/ / www. wheat-expression.com/).

Acknowledgments: This study was supported by the National Natural Science Foundation of China (No. 31671608).

Conflicts of Interest: The authors declare no conflict of interest.

\section{References}

1. Lai, X.; Chahtane, H.; Martin-Arevalillo, R.; Zubieta, C.; Parcy, F. Contrasted evolutionary trajectories of plant transcription factors. Curr. Opin. Plant Biol. 2020, 54, 101-107. [CrossRef]

2. Das, G.M.; Tsiantis, M. Gene networks and the evolution of plant morphology. Curr. Opin. Plant Biol. 2018, 45, 82-87. [CrossRef]

3. Rushton, P.J.; Somssich, I.E.; Ringler, P.; Shen, Q.J. WRKY transcription factors. Trends Plant Sci. 2010, 15, 247-258. [CrossRef]

4. Liebsch, D.; Palatnik, J.F. MicroRNA miR396, GRF transcription factors and GIF co-regulators: A conserved plant growth regulatory module with potential for breeding and biotechnology. Curr. Opin. Plant Biol. 2020, 53, 31-42. [CrossRef] [PubMed]

5. Kim, H.J.; Nam, H.G.; Lim, P.O. Regulatory network of NAC transcription factors in leaf senescence. Curr. Opin. Plant Biol. 2016, 33, 48-56. [CrossRef] [PubMed]

6. Baldoni, E.; Genga, A.; Cominelli, E. Plant MYB transcription factors: Their role in drought response mechanisms. Int. J. Mol. Sci. 2015, 16, 15811-15851. [CrossRef]

7. Jiang, J.; Ma, S.; Ye, N.; Jiang, M.; Cao, J.; Zhang, J. WRKY transcription factors in plant responses to stresses. J. Integr. Plant. Biol. 2017, 59, 86-101. [CrossRef] [PubMed]

8. Thirumalaikumar, V.P.; Devkar, V.; Mehterov, N.; Ali, S.; Ozgur, R.; Turkan, I.; Mueller-Roeber, B.; Balazadeh, S. NAC transcription factor JUNGBRUNNEN1 enhances drought tolerance in tomato. Plant. Biotechnol. J. 2018, 16, 354-366. [CrossRef]

9. Tan, Q.K.; Irish, V.F. The Arabidopsis zinc finger-homeodomain genes encode proteins with unique biochemical properties that are coordinately expressed during floral development. Plant. Physiol. 2006, 140, 1095-1108. [CrossRef]

10. Tran, L.S.; Nakashima, K.; Sakuma, Y.; Osakabe, Y.; Qin, F.; Simpson, S.D.; Maruyama, K.; Fujita, Y.; Shinozaki, K.; YamaguchiShinozaki, K. Co-expression of the stress-inducible zinc finger homeodomain ZFHD1 and NAC transcription factors enhances expression of the ERD1 gene in Arabidopsis. Plant. J. 2007, 49, 46-63. [CrossRef] [PubMed]

11. Hu, W.; DePamphilis, C.W.; Ma, H. Phylogenetic Analysis of the plant-specific zinc finger-homeobox and mini zinc finger gene families. J. Integr. Plant. Biol. 2008, 50, 1031-1045. [CrossRef]

12. Wang, W.; Wu, P.; Li, Y.; Hou, X. Genome-wide analysis and expression patterns of ZF-HD transcription factors under different developmental tissues and abiotic stresses in Chinese cabbage. Mol. Genet. Genomics 2016, 291, 1451-1464. [CrossRef]

13. Windhovel, A.; Hein, I.; Dabrowa, R.; Stockhaus, J. Characterization of a novel class of plant homeodomain proteins that bind to the C4 phosphoenolpyruvate carboxylase gene of Flaveria trinervia. Plant. Mol. Biol. 2001, 45, 201-214. [CrossRef] [PubMed]

14. Ariel, F.D.; Manavella, P.A.; Dezar, C.A.; Chan, R.L. The true story of the HD-Zip family. Trends Plant. Sci. 2007, 12, 419-426. [CrossRef]

15. Krishna, S.S.; Majumdar, I.; Grishin, N.V. Structural classification of zinc fingers: Survey and summary. Nucleic Acids Res. 2003, 31, 532-550. [CrossRef]

16. Khatun, K.; Nath, U.K.; Robin, A.; Park, J.I.; Lee, D.J.; Kim, M.B.; Kim, C.K.; Lim, K.B.; Nou, I.S.; Chung, M.Y. Genome-wide analysis and expression profiling of zinc finger homeodomain (ZHD) family genes reveal likely roles in organ development and stress responses in tomato. BMC Genomics 2017, 18, 695. [CrossRef] [PubMed]

17. Abdullah, M.; Cheng, X.; Cao, Y.; Su, X.; Manzoor, M.A.; Gao, J.; Cai, Y.; Lin, Y. Zinc finger-homeodomain transcriptional factors (ZHDs) in upland cotton (Gossypium hirsutum): Genome-wide identification and expression analysis in fiber development. Front. Genet. 2018, 9, 357. [CrossRef] [PubMed]

18. Liu, M.; Wang, X.; Sun, W.; Ma, Z.; Zheng, T.; Huang, L.; Wu, Q.; Tang, Z.; Bu, T.; Li, C.; et al. Genome-wide investigation of the ZF-HD gene family in Tartary buckwheat (Fagopyrum tataricum). BMC Plant. Biol. 2019, 19, 248. [CrossRef]

19. Perrella, G.; Davidson, M.; O’Donnell, L.; Nastase, A.M.; Herzyk, P.; Breton, G.; Pruneda-Paz, J.L.; Kay, S.A.; Chory, J.; Kaiserli, E. ZINC-FINGER interactions mediate transcriptional regulation of hypocotyl growth in Arabidopsis. Proc. Natl. Acad. Sci. USA 2018, 115, E4503-E4511. [CrossRef] [PubMed] 
20. Park, H.C.; Kim, M.L.; Lee, S.M.; Bahk, J.D.; Yun, D.J.; Lim, C.O.; Hong, J.C.; Lee, S.Y.; Cho, M.J.; Chung, W.S. Pathogen-induced binding of the soybean zinc finger homeodomain proteins GmZF-HD1 and GmZF-HD2 to two repeats of ATTA homeodomain binding site in the calmodulin isoform 4 (GmCaM4) promoter. Nucleic Acids Res. 2007, 35, 3612-3623. [CrossRef]

21. Mayer, K.F.X.; Rogers, J.; Dole El, J.; Pozniak, C.; Eversole, K.; Feuillet, C.; Gill, B.; Friebe, B.; Lukaszewski, A.J.; Sourdille, P.; et al. A chromosome-based draft sequence of the hexaploid bread wheat (Triticum aestivum) genome. Science 2014, 345, 1251788. [CrossRef]

22. Hurst, L.D. The Ka/Ks ratio: Diagnosing the form of sequence evolution. Trends Genet. 2002, 18, 486. [CrossRef]

23. Moore, R.C.; Purugganan, M.D. The early stages of duplicate gene evolution. Proc. Natl. Acad. Sci. USA 2003, 100, 15682-15687. [CrossRef]

24. Lawton-Rauh, A. Evolutionary dynamics of duplicated genes in plants. Mol. Phylogenet Evol. 2003, 29, 396-409. [CrossRef] [PubMed]

25. Liu, X.; Liu, Z.; Niu, X.; Xu, Q.; Yang, L. Genome-wide identification and analysis of the NPR1-like gene family in bread wheat and its relatives. Int. J. Mol. Sci. 2019, 20, 5974. [CrossRef] [PubMed]

26. Simon, M.; Bruex, A.; Kainkaryam, R.M.; Zheng, X.; Huang, L.; Woolf, P.J.; Schiefelbein, J. Tissue-specific profiling reveals transcriptome alterations in Arabidopsis mutants lacking morphological phenotypes. Plant. Cell 2013, 25, 3175-3185. [CrossRef] [PubMed]

27. Abu-Romman, S. Molecular cloning and expression analysis of zinc finger-homeodomain transcription factor TaZFHD1 in wheat. South Afr. J. Bot. 2014, 91, 32-36. [CrossRef]

28. Wang, H.; Yin, X.; Li, X.; Wang, L.; Zheng, Y.; Xu, X.; Zhang, Y.; Wang, X. Genome-wide identification, evolution and expression analysis of the grape (Vitis vinifera L.) zinc finger-homeodomain gene family. Int. J. Mol. Sci. 2014, 15, 5730-5748. [CrossRef] [PubMed]

29. Yong, Y.; Zhang, Y.; Lyu, Y. A Stress-responsive NAC transcription factor from tiger lily (LINAC2) interacts with LIDREB1 and LlZHFD4 and enhances various abiotic stress tolerance in Arabidopsis. Int. J. Mol. Sci. 2019, 20, 3225. [CrossRef]

30. Chen, C.; Chen, H.; Zhang, Y.; Thomas, H.R.; Frank, M.H.; He, Y.; Xia, R. TBtools-An integrative toolkit developed for interactive analyses of big biological data. Mol. Plant. 2020, 13, 1194-1202. [CrossRef]

31. Katoh, K.; Rozewicki, J.; Yamada, K.D. MAFFT online service: Multiple sequence alignment, interactive sequence choice and visualization. Brief. Bioinform. 2019, 20, 1160-1166. [CrossRef]

32. Letunic, I.; Bork, P. Interactive Tree Of Life (iTOL) v4: Recent updates and new developments. Nucleic Acids Res. 2019, 47, W256-W259. [CrossRef] [PubMed]

33. Hu, B.; Jin, J.; Guo, A.; Zhang, H.; Luo, J.; Gao, G. GSDS 2.0: An upgraded gene feature visualization server. Bioinformatics 2015, 31, 1296-1297. [CrossRef]

34. Lescot, M.; Dehais, P.; Thijs, G.; Marchal, K.; Moreau, Y.; Van de Peer, Y.; Rouze, P.; Rombauts, S. PlantCARE, a database of plant cis-acting regulatory elements and a portal to tools for in silico analysis of promoter sequences. Nucleic Acids Res. 2002, 30, 325-327. [CrossRef]

35. Voorrips, R.E. MapChart: Software for the graphical presentation of linkage maps and QTLs. J. Hered 2002, 93, 77-78. [CrossRef] [PubMed]

36. Krzywinski, M.; Schein, J.; Birol, I.; Connors, J.; Gascoyne, R.; Horsman, D.; Jones, S.J.; Marra, M.A. Circos: An information aesthetic for comparative genomics. Genome Res. 2009, 19, 1639-1645. [CrossRef] [PubMed]

37. Wang, Y.; Tang, H.; Debarry, J.D.; Tan, X.; Li, J.; Wang, X.; Lee, T.H.; Jin, H.; Marler, B.; Guo, H.; et al. MCScanX: A toolkit for detection and evolutionary analysis of gene synteny and collinearity. Nucleic Acids Res. 2012, 40, e49. [CrossRef] [PubMed]

38. Ye, J.; Zhang, Y.; Cui, H.; Liu, J.; Wu, Y.; Cheng, Y.; Xu, H.; Huang, X.; Li, S.; Zhou, A.; et al. WEGO 2.0: A web tool for analyzing and plotting GO annotations, 2018 update. Nucleic Acids Res. 2018, 46, W71-W75. [CrossRef]

39. Borrill, P.; Ramirez-Gonzalez, R.; Uauy, C. expVIP: A customizable RNA-seq data analysis and visualization platform. Plant. Physiol. 2016, 170, 2172-2186. [CrossRef] [PubMed]

40. Liu, H.; Xing, M.; Yang, W.; Mu, X.; Wang, X.; Lu, F.; Wang, Y.; Zhang, L. Genome-wide identification of and functional insights into the late embryogenesis abundant (LEA) gene family in bread wheat (Triticum aestivum). Sci. Rep. UK 2019, 9, 13375. [CrossRef] 